\title{
Zusammenwirken von Wissenschaft und Politik als Herausforderung
}

Hansueli Albonico

Dr. med.

Vor 20 Jahren startete das von Bundesrätin Ruth Dreifuss initiierte «Programm Evaluation Komplementärmedizin, PEK», das bis heute international umfassendste Projekt in der Versorgungsforschung zur Komplementärmedizin, mit einem Zeitrahmen von sechs Jahren und einem Budget von sechs Millionen Franken. Nach dem Amtsantritt von Bundesrat Couchepin kam es zu einem jähen vorzeitigen Abbruch des Projektes, welcher jedoch der Volksinitiative «Ja zur Komplementärmedizin» entscheidenden Auftrieb gab: Vor 10 Jahren wurde der Gegenvorschlag zur Initiative mit einer Zweidrittelsmehrheit angenommen. Die umfassende Auswertung der PEK-Resultate fehlt aber seither als verbindende Grundlage für die laufenden wissenschaftlichen, politischen und ökonomischen Diskussionen.

Die Geschichte der Aufnahme der Komplementärmedizin in der Bundesverfassung der Schweiz ist eine spannende Lektion zur Verschränkung von Wissenschaft und Politik. Führen wissenschaftliche Erkenntnisse zu politischen Einstellungen und Vorstössen? Können politische Forderungen durch wissenschaftliche Untersuchungen erfüllt werden? Gewiss ein hochaktuelles Thema, wenn wir z.B. an die Klimadebatte denken.

Im Rahmen der KVG-Revision seit 1996 machte sich die damalige Gesundheitsministerin Ruth Dreifuss für die Grundversorgung stark. Ihr Bekenntnis: «Die GrundversorgerInnen sind die tragende Säule unseres Gesundheitswesens ... Die Entscheidungen, was medizinisch zu tun sei, müssen in der Arzt-Patienten-Beziehung stattfinden ...» Entsprechend setzte sich Dreifuss dafür ein, dass in dem von ihr initiierten «Programm Evaluation Komplementärmedizin, PEK» von Anfang an «Schulmediziner» mitarbeiten, und legte damit den Keim für das spätere Konzept der «Integrativen Medizin».

\section{Wechselnde politische Vorzeichen}

Die politische Vorgabe war mit dem Artikel 32 KVG klar: «Die Leistungen ... müssen wirksam, zweckmässig und wirtschaftlich sein. Die Wirksamkeit muss nach wissenschaftlichen Methoden nachgewiesen werden.» PEK hatte zu allen drei Forderungen die nötigen wis- senschaftlichen Entscheidungsgrundlagen zu erarbeiten, aufgrund deren die ärztlichen komplementärmedizinischen Fachgesellschaften ihre Anträge auf definitive Aufnahme in den Pflichtleistungskatalog der Grundversicherung einreichen sollten. Nach anfänglichen Schwierigkeiten bei der Festlegung der Akteure nahm PEK in den folgenden Jahren laufend an Fahrt auf. In einem Gutachten kam der BAG-Beauftragte Kurt Hess 2014 zum Schluss, «dass hier allerorts in hohem Masse ökonomisch, professionell und effizient gearbeitet worden ist und wird», und bestätigte: «PEK hat allein schon dadurch, dass die historischen Gräben zwischen beiden medizinischen Systemen in schwierigen Konsensverfahren ... überbrückt werden konnten, Einmaliges erreicht, das auch international auf höchste Beachtung gestossen ist.»

Doch nach dem Amtsantritt von Gesundheitsminister Pascal Couchepin wehte dem PEK plötzlich ein eisiger Wind entgegen, welcher an der denkwürdigen Fachtagung vom 21. April 2005 seinen Höhepunkt fand: Im prall gefüllten Auditorium Maximum der Universität Bern sollten nach einer Medienorientierung mit 36 anwesenden Journalisten 17 Referenten die Resultate von PEK vortragen, gemäss der Ausschreibung: «Das PEK ist international bisher das grösste und komplexeste Forschungsprojekt zur komplementärmedizinischen Versorgung der Bevölkerung auf nationaler Ebene.» Jedoch: Von den 17 Referenten traten nur noch vier auf, 
mit nichtssagenden Statements. Drei Tage zuvor war den Referenten auf Anweisung "von oben» ein Redeverbot auferlegt worden, eine Mitarbeiterin wurde von ihrem Institut ausgesperrt, ihr PC beschlagnahmt. "Hinsichtlich der am 21. April stattfindenden Fachtagung PEK ist es Ihnen untersagt, Resultate des Projekts in qualitativer oder quantitativer Hinsicht zu veröffentlichen ... Das Nichteinhalten könnte das Einleiten entsprechender rechtlicher Schritte nötig machen", liess die Programmleitung verlauten. Kurz darauf wurden die Vertreter der Komplementärmedizin sowie das internationale Review-Board vom Schlussbericht ausgeschlossen und die Programmleitung vorzeitig entlassen. Die Politik hatte die Wissenschaft abgewürgt. BR Couchepin sagte im Parlament zwar wenig später: «Je crois qui'il est stupide d'être contre les médecines complémentaires...», unterstellte der Komplementärmedizin aber grundsätzlich Unwissenschaftlichkeit, beschwor eine drohende Mengenausweitung mit «explosivem Potenzial» und schloss die ärztliche Komplementärmedizin wieder aus der Grundversicherung aus.

\section{Wissenschaftlichkeit als beherrschende Frage}

Seither beherrscht die Frage der «Wissenschaftlichkeit» die Auseinandersetzungen zur Komplementärmedizin. Unterdessen hatten aber die Patienten die Anerkennung der Komplementärmedizin selber an die Hand genommen. Bereits zur Jahrhundertwende hatte eine repräsentative Bevölkerungsumfrage der SAMW zur «Neu-Orientierung in der Medizin in der Schweiz» aufgezeigt, dass 69\% der Patienten und Patientinnen «mehr Menschlichkeit», 58\% «mehr Komplementärmedizin», hingegen nur 21\% «mehr Spitzenmedizin» wünschten. Im Erleben der Patienten war die Komplementärmedizin längst eine alltägliche Selbstverständlichkeit, und die meisten hatten, mit einem gewissen Vertrauen in ihre Ärzte, die Erfahrung gemacht, dass Komplementärmedizin durchaus wirksam sein kann. Das 1987 gegründete «Forum Freiheit im Gesundheitswesen, FFG», später "Forum für Ganzheitsmedizin», hatte 1994 unter Federführung des Mitbegründers Herbert Holliger mit massgeblicher Unterstützung durch Nationalrat Rudolf Hafner das Referendum gegen das KVG ergriffen und 2004 zusammen mit weiteren Patientenorganisationen die Volksinitiative «Ja zur Komplementärmedizin» lanciert. Der Rauswurf der ärztlichen Komplementärmedizin aus der sozialen Grundversicherung entwickelte sich zum stärksten Katalysator der Initiative. So kam es 2009 zur denkwürdigen Volksabstimmung für einen neuen Verfassungsartikel 118a: «Bund und Kantone sorgen im Rah- men ihrer Zuständigkeiten für die Berücksichtigung der Komplementärmedizin.»

An den Schweizer Universitäten sorgte dieses klare Verdikt des Volkes indessen für Aufregung. Die Universität Zürich publizierte in der NZZ umgehend ein «Zürcher Manifest zum Nachweis der Wirksamkeit medizinischer Verfahren» mit einem flammenden Aufruf zur ausschliesslichen Gültigkeit des Doppelblindversuchs, mitunterzeichnet von nicht weniger als elf Alt-Dekanen. Zwangsläufig stand somit auch die Administration unter enormem Druck. Unterdessen war das Gesundheitsministerium aber an Bundesrat Burkhalter übergegangen, welcher - wie auch bei all seinen späteren Einsätzen als Aussenminister, etwa in der OECDMission in der Ukraine - ganz auf Vertrauensbildung setzte. Und so geschah denn 2011 «das Wunder von Bern": Mit Hinweis auf die von den Fachgesellschaften bereits zweimal eingereichten und von der ELGK widersprüchlich eingeschätzten Anträge und unter dem Bekenntnis «il ne faut pas réinventer la roue» beschloss Burkhalter die Wiederaufnahme der ärztlichen Komplementärmedizin in der Grundversicherung. Allerdings stellte er diese unter die Bedingung eines erneuten WZW-Nachweises, installierte dazu aber eine direkt dem EDI unterstellte «Begleitgruppe Komplementärmedizin» mit Vertretern der konventionellen und der komplementären Ärzte, der Universitäten und des BAG.

\section{Interdisziplinäre Arbeitsgruppe}

Der Nachfolger im Gesundheitsdepartement, Bundesrat Alain Berset, führte diesen Weg 2012 konsequent weiter. Berset unterstellte den Zulassungsprozess der ärztlichen Komplementärmedizin dem grundsätzlich gültigen «Vertrauensprinzip» und kümmerte sich persönlich um die korrekte Umsetzung der Volksinitiative. Allerdings sollte der übliche Rechtsetzungsprozess noch vier weitere Jahre dauern. Berset hielt sich an sein Bekenntnis: "La situation, après le plébiscite de 2009, est totalement différente», wies jedoch auf die grosse Komplexität des Dossiers hin: «Il s'agit d'un chemin simple, malgré la complexité.» Der Komplexität trug er Rechnung durch die Berufung einer interdisziplinären Arbeitsgruppe mit Vertretern der Schweizerischen Akademie der Medizinischen Wissenschaften (SAMW), der Universitäten, der Krankenversicherer, der «Schulmediziner», der UNION Schweizerischer komplementärmedizinischer Ärzteorganisationen sowie der Schweizerischen Stiftung für Konsumentenschutz SKS. Unter der zielstrebigen Führung durch das BAG erarbeitete dieses Gremium während zweier Jahre die Anpassungen der entsprechenden Verordnungen; am 
16. Juni 2017 wurde der zustimmende Entscheid des Gesamtbundesrates veröffentlicht.

In diesem Prozess spielten nicht zuletzt Publikationen und Gutachten des deutschen Dialogforums «Pluralismus in der Medizin» eine Rolle, welches sich seit seiner Gründung im Jahr 2000 durch den damaligen Präsidenten der Bundesärztekammer, Professor JörgDietrich Hoppe, in zahlreichen Publikationen für die Autonomie des Arztes und einen verbindenden Methodenpluralismus einsetzte. Der neue Artikel 35a KVV basiert denn auch auf Zulassungskriterien, welche die Komplementärmedizin mit der Schulmedizin vereinen, so insbesondere eine dokumentierte Anwendungs- und Forschungstradition, sowohl ausgewiesene wissenschaftliche Evidenz als auch ärztliche Erfahrung und natürlich die spezifische ergänzende Weiterbildung (z.B. Fähigkeitsausweis FMH).

\section{Fehlende Auswertung wissenschaftlicher Erkenntnisse}

Unterdessen war auch das Medizinalberufegesetz (MedBG) angepasst worden; nebst der Betonung der Wahrung des Selbstbestimmungsrechts der Patientinnen und Patienten ist dort explizit die «Vermittlung von angemessenen Kenntnissen über die Methoden und Therapieansätze der Komplementärmedizin" gefordert. Im völlig neu überarbeiteten Schweizerischen Lernzielkatalog «Profiles» wird die Berücksichtigung der Komplementärmedizin insbesondere für die in vielfacher Hinsicht wichtige Anamneseerhebung und für die Erarbeitung einer shared decision verlangt.

In der gesamten Auseinandersetzung um den WZWNachweis in der ärztlichen Komplementärmedizin fehlen uns heute für eine saubere fachliche Debatte die umfassenden Auswertungen der wissenschaftlichen Ergebnisse, welche das PEK mit sechs Millionen Steuerfranken über sechs Jahre erarbeitet hat. Zwar gibt es einige auch qualitativ hochwertige Arbeiten, aber im Verhältnis zur Grösse des Gesamtprojektes sind das wenige.

\section{Begriff "Integrative Medizin» hat sich durchgesetzt}

Allerdings sind wir heute dennoch weiter. In der Schweiz hat sich insgesamt als Ziel die «Integrative Medizin" durchgesetzt, so wie ihn z.B. die American Medical Association (AMA) bereits 2002 in einem Fachartikel unter dem Titel «Integrative Medizin und Systemische Outcome-Forschung» definiert hat: «Kliniker und Forscher benutzen zunehmend den Begriff der 'integrativen Medizin' für das Zusammengehen von Komplementärmedizin mit der konventionellen Biomedizin. Nun ist aber die ledigliche Kombination von Komplementär- und konventioneller Medizin noch nicht ganzheitlich. Integrative Medizin bedeutet ein System höherer Ordnung, welches Gesundheit und Heilung in ihren bio-psycho-sozio-spirituellen Dimensionen erfasst, indem sowohl komplementäre als auch konventionelle Therapieansätze für eine heilsame und wirksame Arzt-Patienten-Beziehung herangezogen werden ...» [1]

\section{Literatur}

1 Bell IR, et al. Integrative Medicine and Systemic Outcome Research: Issues in the Emergence of a New Model for Primary Health Care. Arch Intern Med. 2002;162(2):133-40. doi: 10.1001/archinte.162.2.133.

\section{Das Wichtigste in Kürze}

- Die Geschichte der Aufnahme der Komplementärmedizin in der Bundesverfassung der Schweiz ist eine spannende Lektion zur Verschränkung von Wissenschaft und Politik.

- Vor 20 Jahren startete das von Bundesrätin Ruth Dreifuss initiierte «Programm Evaluation Komplementärmedizin, PEK».

- Im Rahmen des PEK wurden einige qualitativ hochwertige Arbeiten publiziert. Aber im Verhältnis zur Grösse des Gesamtprojektes blieben es wenige.

- In der gesamten Auseinandersetzung um den WZW-Nachweis in der ärztlichen Komplementärmedizin fehlen uns heute für eine saubere fachliche Debatte die umfassenden Auswertungen der wissenschaftlichen Ergebnisse.

\section{L'essentiel en bref}

- L'histoire de l'inscription de la médecine complémentaire dans la Constitution fédérale suisse est une passionnante leçon sur l'interaction entre science et politique.

- Le «Programme d'évaluation des médecines complémentaires» (PEK) a été lancé par la Conseillère fédérale Ruth Dreifuss il y a 20 ans.

- Quelques ouvrages de grande qualité ont été publiés dans le cadre du PEK, mais ils sont restés peu nombreux compte tenu de l'ampleur du projet global.

- Dans l'ensemble de la discussion sur la preuve des critères EAE en médecine complémentaire, il nous manque pour I'instant des analyses complètes des résultats scientifiques pour pouvoir mener un débat technique approprié. 